ISSN 0103-5150

Fisioter. Mov., Curitiba, v. 24, n. 2, p. 221-229 abr./jun. 2011 Licenciado sob uma Licença Creative Commons

\title{
Efeito agudo de diferentes formas de aquecimento sobre a força muscular
}

\author{
Acute effect of different forms of heating on muscle strength
}

\section{Cecilia Voloschen de Albuquerque ${ }^{[a]}$, Juliana Paula Maschio ${ }^{[b]}$, Cristiane Regina Gruber ${ }^{[c]}$, Ricardo Martins de Souza ${ }^{[\mathrm{d}]}$, Sara Hernandez ${ }^{[\mathrm{e}]}$}

[a] Graduanda do oitavo período de Fisioterapia das Faculdades Integradas do Brasil (Unibrasil), Curitiba, PR - Brasil, e-mail: ceciliavoloschen@pop.com.br

[b] Graduanda do oitavo período de Fisioterapia das Faculdades Integradas do Brasil (Unibrasil), Curitiba, PR - Brasil, e-mail: maschio.juliana@gmail.com

[c] Fisioterapeuta e Licenciada em Educação Física, especialista em Treinamento Individual e Qualidade de Vida, Mestre em Engenharia Biomédica, professora da Escola de Saúde das Faculdades Integradas do Brasil (Unibrasil), Curitiba, PR Brasil, e-mail: cristiane_gruber@hotmail.com

[d] Graduado em Educação Física, Mestre em Ciências do Exercício e Esporte, Doutorando em Educação Física, professor da Escola de Saúde das Faculdades Integradas do Brasil (Unibrasil), Curitiba, PR - Brasil, e-mail: profricardo2006@yahoo.com.br

[e] Fisioterapeuta Especialista em Fisioterapia traumato-ortopédica e desportiva pela Pontificia Universidade Católica do Paraná (PUCPR), Mestre em Educação Física, responsável pela avaliação isocinética na Clínica do Joelho, Curitiba, PR Brasil, e-mail: sarinha_hrn@hotmail.com

\section{Resumo}

Os exercícios físicos têm a finalidade de manter e melhorar um ou mais componentes do condicionamento físico, dentre os quais está a força muscular. Para o aperfeiçoamento do treino da força são utilizadas várias técnicas de aquecimento, com vistas a alcançar seus benefícios. 0 objetivo deste estudo foi avaliar a força muscular dos extensores do joelho, antes e após a aplicação de alongamento estático (GAE), exercício aeróbico (GEA) e associação de ambos (GAE+GEA), verificando, dessa maneira, os efeitos dos diferentes tipos de aquecimento sobre a força muscular. Para tanto, participaram do estudo 16 indivíduos do sexo feminino (idade de 22,5 \pm 4,7 anos e IMC de 20,8 $\pm 1,83 \mathrm{~kg} / \mathrm{m} 2$ ), divididos em três grupos (GAE, GEA e GAE+GEA). As variáveis observadas foram: pico de torque concêntrico, pico de torque excêntrico e trabalho total. Cada 
grupo, posteriormente à avaliação da força no dinamômetro isocinético, realizou, após um intervalo mínimo de 48 horas, um protocolo de aquecimento seguido da reavaliação da força muscular. Os resultados obtidos indicaram não haver influência significativa inter e/ou intraprotocolos nas variáveis observadas, considerando nível de significância de $\mathrm{p} \leq 0,05$. Portanto, conclui-se que, de forma aguda, o desempenho de força muscular na extensão do joelho não sofreu alterações significativas após os diferentes protocolos de aquecimento utilizados.

Palavras-chave: Aquecimento. Alongamento muscular. Força muscular. Dinamômetro isocinético.

\section{Abstract}

The physical exercises are designed to maintain and improve one or more components of physical fitness, and among them are muscle strength. For the improvement of strength training, many heating techniques are used in order to achieve its benefits. The aim of this study was to evaluate the muscle strength of knee extensors, before and after applying with static stretching (SSG), aerobic exercise (AEG) and the combination of both $(S S G+A E G)$, checking in this way, the effects of different types of warm on muscle strength. For this participated in the study 16 women (age $22.5 \pm 4.7$ years, BMI $20.8 \pm 1.83 \mathrm{~kg} / \mathrm{m} 2$ ), they were divided into three groups (SSG, AEG and SSG + AEG). The verified variables were: concentric peak torque, eccentric peak torque and total work. Each group, after evaluating of the strength in the isokinetic dynamometer then of minimum 48 hours, performed a warm-up protocol and reviewed the force. The results indicated that there was no significant inter and/or intra protocols on the observed variables, considering a significance level of $p \leq 0.05$. Therefore, we concluded that, acutely, the performance of muscle strength in knee extension did not change significantly after the different protocols used for heating.

Keywords: Warm up. Muscle stretching. Muscle strength. Isokinetic dynamometer.

\section{Introdução}

Os exercícios físicos são movimentos corporais planejados, estruturados e cíclicos, feitos para manter e melhorar um ou mais componentes do condicionamento físico (1). Dentre estes componentes está a força muscular, a qual é definida como a capacidade do músculo esquelético produzir tensão e torque máximos.

Para aperfeiçoar o treinamento de força muscular, são utilizadas várias técnicas de aquecimento, visando a alcançar benefícios como: aumento da temperatura muscular, do metabolismo energético, da elasticidade do tecido conjuntivo, do débito cardíaco, da velocidade de transmissão do impulso nervoso (melhorando a sensibilidade dos proprioceptores, o recrutamento das unidades motoras, a coordenação e a capacidade de suportar carga); diminuição da viscosidade do sistema músculo-tendíneo; redistribuição do fluxo sanguíneo e melhora da difusão do oxigênio disponível nos músculos (2-4).

Dentre as diversas técnicas realizadas previamente a um exercício resistido, e que serão abordadas neste estudo, encontram-se o alongamento e o aquecimento aeróbico. 0 alongamento muscular é frequentemente efetuado nas práticas desportivas, com o objetivo de aumentar a flexibilidade muscular e a amplitude articular, assim como, possivelmente, melhorar o desempenho atlético (5). Já os exercícios aeróbicos tendem a aumentar a temperatura corporal, possibilitando maior velocidade das reações químicas no corpo humano (6).

Embora a literatura evidencie os benefícios do aquecimento, ainda surgem divergências quanto ao tipo de aquecimento que seria mais eficiente para melhorar o desempenho nos exercícios resistidos (7). 0 treinamento da flexibilidade realizado antes desses exercícios vem trazendo controvérsias no âmbito científico no que se refere aos seus efeitos para a performance muscular do indivíduo (8). Pesquisas comprovam que o alongamento pode prevenir encurtamentos teciduais, otimizando o desenvolvimento de contrações voluntárias máximas e o desempenho muscular, o que contribuiria para o treino de força e potência $(9,10)$. Porém, estudos apontam que a realização de exercícios de alongamento antes 
das atividades que envolvam força acarreta prejuízo ao desempenho desta (11-15). Entretanto, alguns autores não observaram efeitos deletérios dos exercícios de flexibilidade e de outras formas de aquecimento sobre a força muscular (16-18).

Sendo assim, o objetivo deste estudo foi avaliar a força muscular dos extensores do joelho, antes e após a aplicação de aquecimento com alongamento estático, exercício aeróbico e associação de ambos, verificando, dessa maneira, os efeitos dos diferentes tipos de aquecimento sobre a força muscular.

\section{Materiais e métodos}

\section{Casuística}

Foram selecionados para este estudo 18 indivíduos do sexo feminino, porém dois deles foram excluídos por apresentarem dor durante a realização dos testes, resultando em uma amostra constituída por 16 participantes $(22,5 \pm 4,7$ anos, $162 \pm 6,13 \mathrm{~cm}$, $54,89 \pm 7,26 \mathrm{~kg}$ e $20,8 \pm 1,83 \mathrm{~kg} / \mathrm{m}^{2}$ ). Os critérios de inclusão foram: a) serem classificados como ativos ou irregularmente ativos por meio do Questionário Internacional de Atividade Física (IPAQ) versão curta; b) não apresentarem lesões osteomioarticulares que dificultassem a realização da avaliação e a aplicação do programa de exercícios.

Todos os voluntários foram informados sobre os objetivos e procedimentos do estudo e assinaram um Termo de Consentimento Livre e Esclarecido. 0 estudo foi aprovado pelo Comitê de Ética das Faculdades Integradas do Brasil (Unibrasil), com parecer n. 025/ 2010, e está de acordo com a Resolução n. 196/96 de pesquisa em seres humanos, do Conselho Nacional de Saúde.

\section{Procedimentos}

Todos os procedimentos foram realizados nas dependências da Clínica do Joelho (Curitiba, PR), com o auxílio da fisioterapeuta da clínica e colaboradora da pesquisa, da seguinte maneira:

Objetivando reduzir a margem de erro durante a realização das avaliações, foram adotadas as seguintes estratégias: a) instruções padronizadas sobre rotina de exercícios e coleta de dados foram oferecidas antes de cada procedimento; b) o avaliador estava
Tabela 1 - Procedimentos

\begin{tabular}{ll}
\hline Dia $\mathbf{1}$ & Dia 2 \\
\hline Familiarização & Aquecimento \\
Teste de FCVM* de & Reavaliação \\
extensão do joelho & \\
\hline
\end{tabular}

Legenda: * = teste de força de contração voluntária máxima.

atento quanto à posição adotada pelo praticante no momento da avaliação; c) cada procedimento foi orientado e/ou executado por um único avaliador.

Todas as avaliações da força de contração voluntária máxima (FCVM) da musculatura extensora do joelho foram realizadas no dinamômetro isocinético (CYBEX - modelo NORM 7000). Somente o membro dominante foi avaliado. A descrição das etapas do estudo encontra-se a seguir:

\section{Avaliação da FCVM de extensão do joelho} sem aquecimento prévio

Primeiramente, a dominância do membro inferior foi identificada. 0 eixo de rotação do dinamômetro foi alinhado com o eixo anatômico do joelho (epicôndilo lateral do fêmur), enquanto que o braço de alavanca do dinamômetro foi fixado na região distal da perna ( $5 \mathrm{~cm}$ acima do maléolo medial), de forma a permitir um arco completo de dorsiflexão do tornozelo. A amplitude da angulação do teste foi limitada em $90^{\circ}$ com início em $0^{\circ}$ de extensão e término em $90^{\circ}$ de flexão do joelho (19), sendo feita a correção da gravidade como parte dos procedimentos de preparo do dinamômetro $(20,21)$. 0 encosto da cadeira do dinamômetro foi ajustado a um ângulo de $120^{\circ}$, a fim de aumentar a inclinação do quadril e, por conseguinte, aumentar o alongamento do músculo reto femoral e diminuir a coativação dos músculos isquiotibiais (22), fazendo assim com que haja maior produção de força do quadríceps femoral (22). Durante a avaliação, os sujeitos receberam orientação para segurarem firmemente nos apoios laterais do assento, auxiliando a realização da força durante o movimento contrarresistido (Figura 1). Este foi executado a uma velocidade angular de $30 \%$ s - considerada lenta, segundo Terreri et al. (23) -, usada para aquisição de maior produção de força $(21,23,24)$. 
Em seguida, executou-se um período de familiarização ao equipamento, que incluiu a realização de três contrações submáximas dos extensores de joelho (25), a fim de possibilitar o aprendizado do movimento com o funcionamento da máquina e o recrutamento de unidades motoras (26). Após a familiarização aguardou-se 45 segundos para a avaliação inicial, na qual o indivíduo realizou uma contração máxima (fase concêntrica e excêntrica). Segundo Dvir (25), um intervalo entre as séries de 30 a 60 segundos é suficiente para recuperação muscular e desempenho ideal na sequência do teste. Neste momento, foram coletadas e registradas, por meio do dinamômetro, as seguintes variáveis isocinéticas: pico de torque concêntrico (PTC), pico de torque excêntrico (PTE) e trabalho total (TT).

\section{Aquecimento}

Após um período mínimo de 48 horas $(27,28)$ da avaliação inicial, os sujeitos foram submetidos a um programa de aquecimento, sendo selecionados aleatoriamente para fazerem parte de um dos três grupos - alongamento estático (GAE), exercício aeróbico (GEA) ou exercício aeróbico mais alongamento estático (GAE+GEA).

Os voluntários participantes do grupo GAE (seis indivíduos) foram submetidos ao alongamento passivo do músculo quadríceps. Após serem orientados, foram posicionados em decúbito dorsal com os membros inferiores para fora da maca. Inicialmente, o membro não dominante deveria permanecer em flexão de quadril e joelho, sendo mantido pelo participante em

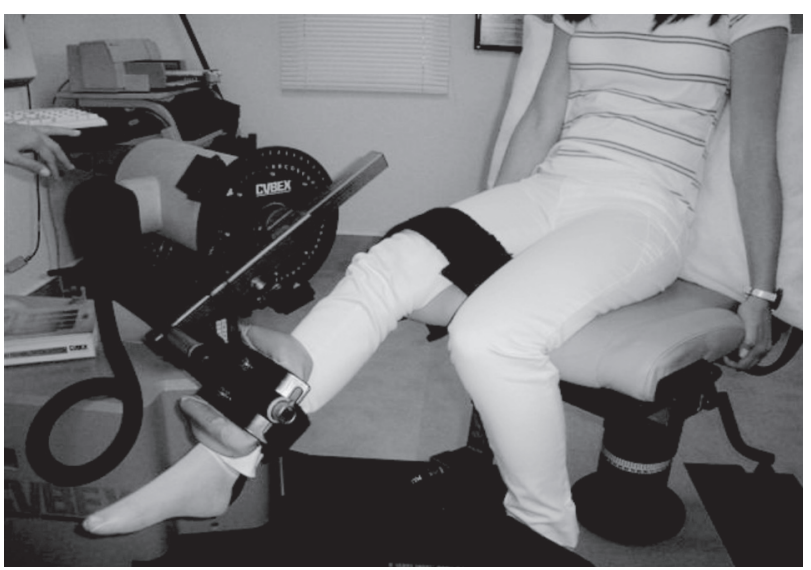

Figura 1 - Posicionamento do voluntário na cadeira do dinamômetro isocinético direção ao tórax (abraçando o membro) enquanto o membro dominante, com auxílio do avaliador, era posicionado em extensão de quadril e flexão de joelho até o limite no qual o participante referisse estar sentindo tensão máxima suportável de alongamento da musculatura extensora de joelho, porém sem sentir dor. Neste momento, o alongamento foi mantido durante 30 segundos (29). Após um intervalo de 1 minuto, o procedimento foi repetido no mesmo membro (20). Realizaram-se quatro repetições em cada membro inferior (29) de maneira alternada.

Os seis indivíduos que participaram do grupo GEA realizaram o aquecimento na bicicleta ergométrica (Ergofit $^{\circledR}$, modelo 167). Foram posicionados e orientados pelo avaliador, da seguinte maneira: a altura do banco foi definida com base na altura da espinha ilíaca ântero-superior do voluntário e a distância do banco para o guidom ajustada com base na flexão do tronco em aproximadamente 30 graus (28). 0 exercício foi realizado à velocidade entre 60 e $70 \mathrm{rpm}(30)$, com carga de $50 \mathrm{~W}$ (30), durante 10 minutos (28).

Para o grupo GAE+GEA foram realizados os mesmos exercícios do grupo GAE e GEA, seguidamente um ao outro, com intervalo de um minuto entre o alongamento estático e o exercício aeróbico. Este grupo foi constituído de quatro participantes, pois dois foram excluídos do estudo por apresentarem dor durante a realização dos testes.

\section{Reavaliação da FCVM de extensão do joelho após aquecimento prévio}

Um minuto após o aquecimento, os sujeitos foram reavaliados no dinamômetro isocinético, seguindo os mesmos parâmetros da avaliação inicial. Para comparação da força da musculatura extensora de joelho sem e com aquecimento prévio, foram coletadas e registradas as seguintes variáveis isocinéticas: PTC, PTE e TT. Os resultados de pico de torque foram extraídos do melhor ângulo de produção de força dado pelo gráfico produzido pelo dinamômetro, e o trabalho total foi obtido pela soma do trabalho concêntrico e do trabalho excêntrico de 1-RM.

\section{Análise estatística}

O teste de Kolmogorov-Smirnof foi aplicado para confirmar a normalidade dos dados. Assumindo-se 
uma distribuição normal, um número de análises de variância ANOVA multifatorial com medidas repetidas foi aplicado para testar as diferenças entre os grupos experimentais GAE, GEA e GAE+GEA. 0 teste de Tukey foi aplicado quando houve diferença entre os grupos para determinar onde as diferenças ocorreram. Os testes estatísticos para todas as variáveis analisadas apresentaram nível de significância de $\mathrm{p} \leq 0,05$ e foram aplicados por meio do software Statística ${ }^{\circledR}$ versão 7.0.

\section{Resultados}

O presente estudo objetivou avaliar a força muscular dos extensores do joelho, antes e após a aplicação de aquecimento com alongamento estático, exercício aeróbico e a associação de ambos. Para tanto, analisou-se o PTC, o PTE e o TT antes e depois dos diferentes protocolos aplicados.

As Figuras 2, 3 e 4 apresentam, respectivamente, a média e o desvio-padrão do PTC, PTE e TT, antes e depois do alongamento estático, do exercício aeróbico e da associação de ambos. Observa-se que as diversas técnicas realizadas previamente ao exercício resistido, neste estudo, não produziram diferenças estatisticamente significativas inter e/ou intraprotocolos nas variáveis analisadas ( $\mathrm{p} \leq 0,05$ ), ou seja, não houve alteração do desempenho de força muscular.

\section{Discussão}

Neste estudo, a força muscular de extensão do joelho foi avaliada antes e após a aplicação de alongamento estático, aquecimento aeróbico e associação de ambos, a fim de avaliarem-se os efeitos dos diferentes tipos de aquecimento sobre a força muscular. De forma geral, os resultados indicaram não haver influência significativa inter e/ou intraprotocolos nas variáveis observadas (PTC, PTE e TT).

Estes resultados assemelham-se aos de Amaral et al. (16), que verificaram a influência do alongamento no desempenho da força de membros inferiores no teste de 1 RM em atletas. Para tanto, após a familiarização, os indivíduos, em uma sessão, realizavam o aquecimento em bicicleta durante 10 minutos mais o alongamento estático, e na sessão seguinte, realizavam o aquecimento somente na bicicleta. Após cada sessão a carga máxima foi reavaliada e concluiu-se que os diferentes aquecimentos aplicados não influenciaram

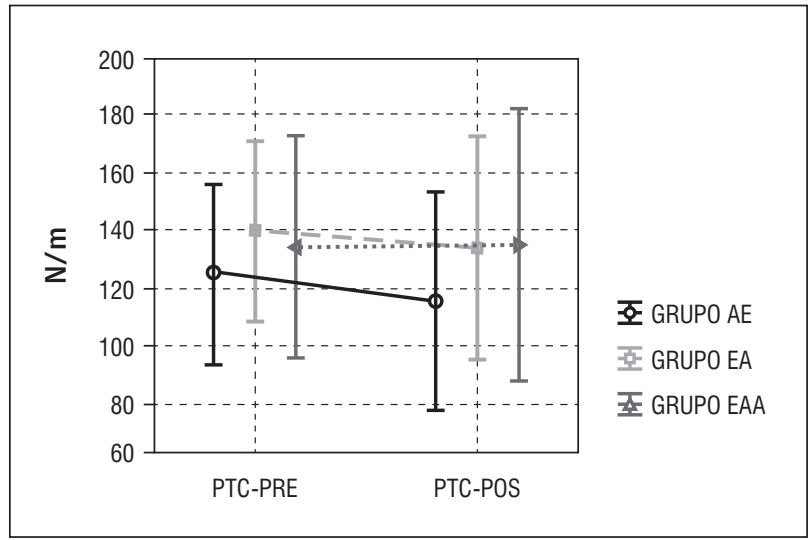

Figura 2 - Média e desvio-padrão do PTC, dado em Newton por metro (N/m), antes e depois da aplicação de alongamento estático (AE), do exercício aeróbico (EA) e do alongamento estático mais exercício aeróbico (EAA)

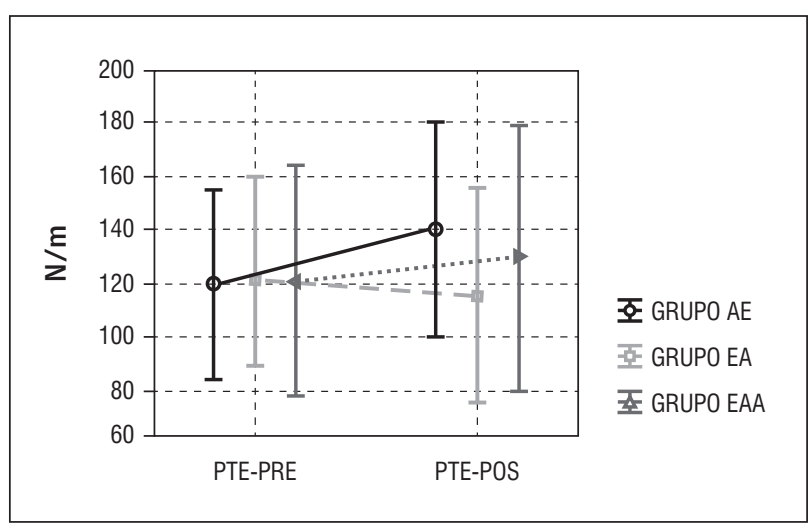

Figura 3 - Média e desvio-padrão do PTE, dado em Newton por metro $(\mathrm{N} / \mathrm{m})$, antes e depois da aplicação de alongamento estático (AE), do exercício aeróbico (EA) e do alongamento estático mais exercício aeróbico (EAA)

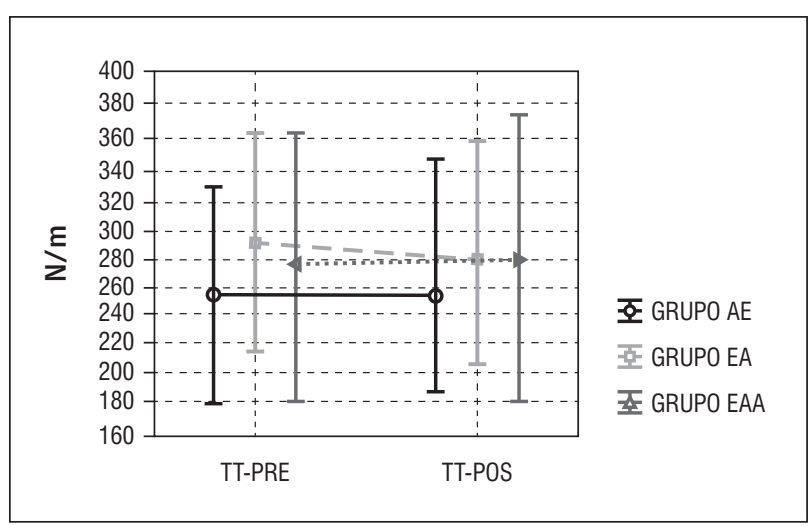

Figura 4 - Média e desvio-padrão do TT, dado em Joules (J), antes e depois da aplicação de alongamento estático $(A E)$, do exercício aeróbico (EA) e do alongamento estático mais exercício aeróbico (EAA) 
negativamente o desempenho de $1 \mathrm{RM}$. Este achado, apesar de amostra e método de avaliação distintos, corrobora os resultados do estudo em questão.

Em revisão de literatura realizada por Shrier et al. (11), com o objetivo de avaliar se o alongamento proporciona melhorias no desempenho, verificou-se que 22 dos 23 artigos analisados sugeriram não haver nenhum tipo de benefício para a força isométrica, torque isocinético ou para o salto em altura. Exemplificando este resultado, pode-se observar que no presente estudo o alongamento também não foi capaz de alterar as variáveis relacionadas à força muscular.

0 estudo de Fermino et al. (31), com 12 voluntários, observou não haver diferença significativa no número máximo de repetições em cada série com carga para $10 \mathrm{RM}$ no exercício de mesa flexora, independentemente da forma de aquecimento realizado: alongamento por meio do método passivo estático ou aquecimento específico. Isso demonstra que aquecimentos gerais, como alongamentos e exercícios que envolvem o corpo como um todo, podem apresentar alguns benefícios, mas não são tão eficazes para favorecer o ganho de força (31). 0 mesmo ocorreu no estudo em questão, porém utilizando alongamento estático e exercício aeróbico.

Outros estudos que não verificaram alterações significantes na capacidade de gerar força após diferentes protocolos de aquecimento foram o de Simão et al. (28) e Nader et al. (32), que analisaram o aquecimento específico, aeróbico e de flexibilidade passiva, e o de Nicoli et al. (7), que avaliaram o aquecimento específico e o aeróbico.

Em contrapartida, Souza et al. (33), ao analisarem o efeito agudo do intervalo passivo e do alongamento estático no desempenho de séries múltiplas dos exercícios de agachamento e supino reto, observaram que pode haver diminuição do número de repetições máximas quando se utiliza, entre as séries, o exercício de alongamento. Corroborando esse estudo, Tricoli e Paulo (34) determinaram os efeitos de uma sessão aguda de exercícios de alongamento estático no desempenho de tarefa que envolve força máxima no aparelho de leg press e verificaram uma redução expressiva na carga média obtida no teste. O estudo comparou o teste de carga máxima precedido por uma sessão de alongamento para membros inferiores com duração de 20 minutos com outra sessão na qual o mesmo teste foi precedido por um aquecimento especifico que consistia em cinco repetições com intensidade igual a 50\% $1 \mathrm{RM}$.
Fowles et al. (35) avaliaram a performance da força muscular após 30 minutos de alongamento passivo máximo. Os resultados desse estudo evidenciaram a diminuição da força, em comparação ao grupo controle. De acordo com os autores, o responsável pela queda na capacidade de força máxima após exercícios de alongamento pode ser o decréscimo na ativação das unidades motoras.

Na mesma linha de pensamento, Wilson et al. (36) apontam que o alongamento estático reduziria a tensão passiva e a rigidez da musculatura esquelética, tornando a unidade músculo-tendínea mais maleável, a qual passaria por um rápido período de diminuição de comprimento, com ausência de sobrecarga, até que os componentes elásticos do sistema fossem ajustados o suficiente para a transmissão da força, colocando um componente contrátil numa posição menos favorável em termos de produção de força nas curvas de força-comprimento e força-velocidade. Outra explicação para o decréscimo de força após o alongamento é relacionada a fatores mecânicos, que provocariam modificações plásticas, tanto nos componentes elásticos dos tecidos moles como na fáscia muscular, induzindo modificações mais permanentes em seus comprimentos (31).

Deve-se levar em consideração que no presente estudo o método de alongamento estático foi realizado em apenas uma sessão, e após um minuto de intervalo reavaliou-se a contração voluntária máxima de extensão do joelho. Sugere-se, dessa maneira, que o tempo de estimulação empregado (120 segundos, divididos em quatro séries de 30 segundos) foi insuficiente para alterar fisiologicamente a estrutura muscular. Portanto, seguindo-se o raciocínio de Fowles (35) e Wilson (36), a não alteração nas variáveis analisadas (PTC, PTE e TT) pode ser decorrente da interferência de fatores como intensidade e volume dos tipos de aquecimento empregados. No entanto, Depino et al. (37), ao analisarem a duração do efeito agudo do alongamento dos isquiotibiais após o término de um protocolo realizado com quatro séries de 30 segundos, verificaram a manutenção do comprimento muscular por três minutos. Por conseguinte, de acordo com os autores, as modificações do comprimento muscular ainda estariam presentes após um minuto do alongamento (tempo utilizado neste estudo para reavaliação da força).

Além do alongamento estático, alguns estudos $(38,39)$ explanam que os exercícios aeróbicos, quando realizados previamente aos exercícios 
resistidos, exercem redução no desempenho da força. Uma possível justificativa para tal fato é que uma sessão de exercício de resistência promoveria mudanças metabólicas agudas durante o treino de força subsequente (40), comprometendo as adaptações decorrentes ao estímulo dessa força a partir da alteração do padrão de recrutamento muscular (40). Sabe-se ainda que fadiga, intensidade e volume de aquecimento são fatores decisivos ao se analisar a performance muscular, parecendo existir um nível ótimo dessas variáveis durante o aquecimento, que estaria relacionado a um melhor rendimento (3). No entanto, cabe ressaltar que, de acordo com esses estudos $(38,39)$, os exercícios de resistência de longa duração (21-160 minutos) são os que poderiam influenciar negativamente a prática de exercícios resistidos. Considerando que no estudo em questão o tempo total de treino aeróbico foi de 10 minutos, conclui-se que a realização de exercícios aeróbicos com baixo volume e intensidade, antes do treino de força, não é capaz de produzir efeitos deletérios no desempenho muscular.

Outro fator que pode ter influenciado os resultados do estudo é a ocorrência da familiarização e do teste de contração voluntária máxima de extensão do joelho no mesmo dia, pois pode ter ocorrido fadiga muscular, comprometendo a eficácia na realização do teste inicial. Do mesmo modo, fatores de cunho psicológico, como motivação para execução dos testes e habituação, devem ser considerados como relevantes ao se avaliar o desempenho muscular em voluntários. Ainda, para uma maior fidedignidade dos resultados após os diferentes tipos de aquecimento, haveria a possibilidade da amostra de cada grupo ser constituída de maior número de indivíduos, ou os participantes do estudo poderiam ter executado todos os tipos de aquecimento propostos em diferentes dias e reavaliados após cada sessão. Apesar desses possíveis vieses, pode-se considerar que os cuidados preparatórios realizados antes da execução das medidas de avaliação e durante as três formas de aquecimento foram suficientes para garantir a validade interna do estudo.

\section{Conclusão}

De forma aguda, assumindo-se as limitações deste estudo e as características do grupo amostral, infere-se, com base nos resultados, que não existiram diferenças estatisticamente significativas em relação ao PTC, PTE e TT antes e depois dos diferentes protocolos de aquecimento utilizados, não alterando assim o desempenho de força muscular na extensão do joelho avaliada por meio do dinamomêtro isocinético. Isto posto, sugere-se como aplicação prática, que a parte inicial de uma sessão de testes de força ou treinamento resistido - o aquecimento seja realizada de acordo com o objetivo do trabalho (treinamento ou reabilitação) e conforme a adaptação de cada indivíduo. Contudo, novos estudos devem ser efetuados, utilizando-se maiores níveis de volume e intensidade de aquecimento, maior amostra e voluntários de genêro, com faixa etária e nível de condicionamento físico distintos.

\section{Referências}

1. Silva A, Macri SPCS. Avaliação da qualidade de vida e flexibilidade de mulheres climatéricas após alongamento muscular. Revista PIBIC. 2007;4(1):71-80.

2. Bishop D. Warm up I: potential mechanisms and the effects of passive warm up on exercise performance. Sports Med. 2003;33(6):439-54.

3. Bishop D. Warm up II: performance changes following active warm up and how to structure the warm up. Sports Med. 2003;33(7):483-98.

4. Young WB, Behm DG. Should static stretching be used during a warm-up for strength and activities? Stren Cond J. 2002;24(6):33-37.

5. Endlich PW, Farina GR, Dambroz C, Gonçalves WLS, Moysés MR, Mill JG, et al. Efeitos agudos do alongamento estático no desempenho da forca dinâmica em homens jovens. Rev Bras Med Esporte. 2009; 15(3):200-3.

6. Robergs A, Robergs SO. Fisiologia do exercício. São Paulo: Phorte; 2002.

7. Nicoli AIV, Cordova KO, Barreto, ACLYG, Novaes JS. Influência dos diferentes tipos de aquecimento no número de repetições nos exercícios resistidos. Arq Mov. 2007;3(2):42-55.

8. Ramos GV, Santos RR, Gonçalves A. Influência do alongamento sobre a força muscular: uma breve revisão sobre as possíveis causas. Rev Bras Cineantropom Desempenho Hum. 2007;9(2):203-4. 
9. Batista LH, Camargo PR, Oishi J, Salvini TF. Efeitos do alongamento ativo excêntrico dos músculos flexores do joelho na amplitude de movimento e torque. Rev Bras Fisioter. 2008;12(3):176-82.

10. Kokkonen J, Nelson AG, Cornwell C. Acute muscle stretching inhibits maximal strength performance. Med Sci Sports Exerc. 1998;69(4):411-15.

11. Shrier I. Does stretching improve performance? A systematic and critical review of the literature. Clin J Sport Med. 2004;14(5):267-73.

12. Cramer JT, Housh TJ, Johnson GO, Miller JM, Coburn JW, Beck TW. Acute effects of static stretching on peak torque in women. J Strength Cond Res. 2004; 18(2):236-41.

13. Cramer JT, Housh TJ, Johnson GO, Coburn JW, Beck TH. The acute effects of static stretching on peak torque, mean power output, electromyography, and mechanomyography. Eur J Appl Physiol. 2005;93(5-6): 530-9.

14. Marek SM, Cramer JT, Fincher AL, Massey LL, Dangelmaier SM, Purkayastha S, et al. Acute effects of static and proprioceptive neuromuscular facilitation stretching on muscle strength and power output. J Athl Train. 2005;40(2):94-103.

15. Power K, Behem D, Cahill F, Carroll M, Young W. An acute bout of static stretching: effects on force and jumping performance. Med Sci Sports Exerc. 2004;36(8):1389-96.

16. Amaral PR, Araújo SR, Chagas MH. Stretching exercises used to warm up do not improve 1RM performance of volleyball players. Proceedings of the 25 ISBS Symposium; 25-29 jun. 2007; Ouro Preto: Universidade Federal de Ouro Preto; 2007.

17. Egan AD, Cramer JT, Massey L, Marek SM. Acute effects of static stretching on peak torque and mean power output in national collegiate athletic association division I women's basketball players. J Strength Cond Res. 2006;20(4):778-82.

18. Yamaguchi T, Ichii K. Effects of static stretching for 30 seconds and dynamic stretching on leg extension power. J Strength Cond Res. 2005;19(3):677-83.

19. Rebelo NA, Oliveira J. Relação entre a velocidade, a agilidade e a potência muscular de futebolistas profissionais. Rev Port Cien Desp. 2006;6(3):342-48.
20. Grego NA, Manffra EF. Influência do volume de alongamento estático dos músculos isquiotibiais nas variáveis isocinéticas. Rev Bras Med Esporte. 2009; 15(2):104-9.

21. D’Alessandro RL, Silveira EAP, Anjos MTS, Silva AA, Fonseca ST. Análise da associação entre a dinamometria isocinética da articulação do joelho e o salto horizontal unipodal, hop teste, em atletas de voleibol. Rev Bras Med Esporte. 2005;11(5):271-7.

22. Lima CS, Pinto RS. Cinesiologia e musculação. Porto Alegre: Artmed; 2006.

23. Terreri ASAP, Greve JMD, Amatuzzi MM. Avaliação isocinética no joelho do atleta. Rev Bras Med Esporte. 2001;7(5):170-4.

24. Bittencourt NFN, Amaral GM, Anjos MTS, D’Alessandro R, Silva AA, Fonseca ST. Avaliação muscular isocinética da articulação do joelho em atletas das seleções brasileiras infanto e juvenil de voleibol masculino. Rev Bras de Med Esporte. 2005;11(6):331-6.

25. Dvir Z. Isocinética: avaliações musculares, interpretações e aplicações clínicas. São Paulo: Manole; 2002.

26. Perrin DH. Isokinetic exercise and assessment. Champaingn (IL/USA): Human Kinetics; 1993.

27. Cardozo G, Torres JB, Dantas EHM, Simão R. Comportamento da força muscular após o alongamento estático. Rev Treinamento Desportivo. 2006;7(1):73-6.

28. Simão R, Senna G, Leitão N, Arruda R, Priore M, Maior AM, et al. Influência dos diferentes protocolos de aquecimento na capacidade de desenvolver carga máxima no teste de 1RM. Fit Perf J. 2004; 3(5):261-5.

29. Bandy WD, Iron JM, Briggler M. The effect of time and frequency of static streching on flexibility of the hamstring muscles. Phyr Thu. 1997;77(10):1090-96.

30. Brentano MA, Rodrigues LP, Kruel LFM. Efeitos de diferentes sessões de aquecimento no torque e amplitude articular de homens jovens. Rev Bras Educ Fís Esp. 2008;22(1):53-62.

31. Fermino RC, Winiarski ZH, Rosa RJ, Lorenci RG, Buso S, Simão R. Influência do aquecimento específico e de alongamento no desempenho da força muscular em 10 repetições máximas. Rev Bras Ci e Mov. 2005;13(4):25-32. 
32. Nader AN, Silva AMG, Rocha HNB, Chaves CPG, Miranda H, Simão R, et al. Influência dos aquecimentos geral e específico na força de membros superiores. Rev Bras Presc Fisiolog Exerc. 2009; 3(18):517-21.

33. Souza ACR, Bastos CLB, Portal MND, Salles BF, Gomes TM, Novaes JS. Efeito agudo do intervalo passivo e do alongamento no desempenho de séries múltiplas. Rev Bras Cineantropom Desempenho Hum. 2009;11(4):435-43.

34. Tricoli V, Paulo AC. Efeito agudo dos exercícios de alongamento sobre o desempenho de força máxima. Rev Ativ Fis e Saúde. 2002;7:6-13.

35. Fowles JR, Sale DG, MacDougall M. Reduce strength after passive stretch of the human plantar flexores. J Appl Physol. 2000;89(11):79-88.

36. Wilson GJ, Murphy AJ, Pryor JF. Muscle tendinous stiffness: its relationship to eccentric, isometric, and concentric performance. Can J Appl Physiol. 1994;76(27):14-9.

37. Depino GM, Webright WG, Arnold BL. Duration of maintained hamstring flexibility after cessation of an acute static stretching protocol. J Athl Train. 2000; 35(1):56-9.
38. Haff GG, Stone MH, Warren BJ, Keith R, Johnson RL, Nieman DC, et al. The effect of carbohydrate supplementation on multiple sessions and bouts of resistance exercise. J Strength Cond Res. 1999;13(1):112-7.

39. Schell TC, Wright G, Martino P, Ryder J, Craig BW. Postexercise glucose, insulin, and c-peptide responses to carbohydrate supplementation: running vs. resistance exercise. J Strength Cond Res. 1999; 13(2): 372-80.

40. Aoki MS, Pontes Jr FL, Navarro F, Uchida MC, Bacurau RFP. Suplementação de carboidrato não reverte o efeito deletério do exercício de endurance sobre o subsequente desempenho de força. Rev Bras Med Esporte. 2003;9(5):282-7.

Recebido: 18/10/2010

Received: 10/18/2010

Aprovado: 18/03/2011

Approved: 03/18/2011 